\title{
Restricted Full Three-Body Problem: Application to Binary System 1999 KW4
}

\author{
Julie Bellerose* $*$ and Daniel J. Scheeres $\ddagger$ \\ University of Michigan, Ann Arbor, Michigan 48109
}

DOI: $10.2514 / 1.30937$

\begin{abstract}
The motion of a satellite in the gravitational field of a binary system is investigated. The two bodies of the binary system are modeled as a sphere and a triaxial ellipsoid and the satellite is assumed to have no influence on the motion of the two primaries. The case of relative equilibria in the full two-body problem is assumed. In earlier papers, we looked at equilibrium solutions in the restricted full three-body problem, especially for $L_{4,5}$. In the present work, we investigate energy constraints on the motion of the spacecraft using zero-velocity curves, collinear Lagrangian points, and the Jacobi constant for varying parameters of the system. We study transit and nontransit trajectories between the components. The methods are applied in the study of the binary asteroid system $1999 \mathrm{KW} 4$, and some results are shown.
\end{abstract}

\section{Introduction}

$\mathbf{I}^{\mathrm{s}}$ N RECENT years, we have witnessed a few spacecraft missions sent to small bodies of our solar system. With the growing interest in asteroid systems in the scientific community, it is fair to assume that a mission will target a binary asteroid system in the near future. To develop a model suitable for binary asteroid systems, we took into account the mass distribution of one of the bodies of the restricted three-body problem (R3BP), and named it the restricted full threebody problem (RF3BP). The "full" problem has proven to be a dynamically rich problem.

Previous papers have considered relative equilibria of the binary itself, or the full two-body problem (F2BP) $[1,2]$ and stability of equilibrium solutions in the RF3BP [3,4]. In [1], the author discusses the stability region of the relative equilibria for an ellipsoid-sphere system; it is common to find systems made of a small ellipsoid and a large sphere [5]. Scheeres [2] gives a more general discussion on the conditions for equilibria in the general case of the F2BP under sphere restriction. In the case of the RF3BP, [3] derived the general equations of motion of a particle in the RF 3 BP, whereas Bellerose and Scheeres [4] looked at equilibrium solutions for an ellipsoidsphere system. In particular, the stability of the equilateral Lagrangian points have been investigated. It was found that the ellipsoid reduces the stability region compared with the R3BP.

In the present work, we give a more general discussion of the equilibrium solutions and the energy constraints associated with them for an ellipsoid-sphere system. In the case of an equilibrium in the F2BP, the system allows for one integral of motion, the Jacobi integral. As for the R3BP, different values of the Jacobi integral lead to the definition of the Lagrangian points, in which the regions of allowable motion can be mapped. For particular values of the free parameters of the system, we solve for the collinear Lagrangian points. Because the mass distribution of one of the bodies is taken into account, the system has an extra physical constraint in that Lagrangian points should be located outside of the body.

Presented as Paper 224 at the AAS Spaceflight Mechanics Meeting, Sedona, Arizona, 28 January-1 February 2007; received 14 March 2007; revision received 1 August 2007; accepted for publication 1 August 2007. Copyright $\odot 2007$ by the American Institute of Aeronautics and Astronautics, Inc. All rights reserved. Copies of this paper may be made for personal or internal use, on condition that the copier pay the $\$ 10.00$ per-copy fee to the Copyright Clearance Center, Inc., 222 Rosewood Drive, Danvers, MA 01923; include the code 0731-5090/08 \$10.00 in correspondence with the CCC.

*Ph.D. Candidate, Department of Aerospace Engineering. Student Member AIAA.

${ }^{\dagger}$ Associate Professor, Department of Aerospace Engineering; scheeres@ umich.edu. Associate Fellow AIAA.
Looking at the case of $L_{1}$ especially, if the zero-velocity curves are open, this implies that particles could transit from one body to the other. This problem has interesting application to surface vehicles transiting between the binary components. As rovers are now sent to investigate small bodies to get samples back to Earth, such as the JAXA's Hayabusa mission [6], surface motion on both bodies and transfers between them are important to consider in the design of missions to a binary system. In this case, however, because of the unstable nature of $L_{1}$, only specific regions and conditions at the body surface can lead to transit or nontransit trajectories. The initial and final conditions at the surface are crucial for designing transfer maneuvers of a vehicle. These findings can also give us a better understanding of particles dynamics near the surface.

In the following sections, we first review the dynamics of the binary system itself before looking at the motion of a particle in this gravitational field in more detail. Finally, we apply these theoretical developments to a recently analyzed binary system, designated Asteroid 1999 KW4 [ㄷ].

\section{Full Two-Body Problem}

Before defining the dynamics for a particle in orbit about a binary system, we need to address the full two-body problem itself. We consider an ellipsoid-sphere system. The general problem is represented in Fig. 1. In this model, $M_{1}$ is the mass of the spherical shape and $M_{2}$ is the mass of the ellipsoid. The mass ratio of the two primaries is defined as

$$
v=\frac{M_{1}}{M_{1}+M_{2}}
$$

We use $\boldsymbol{r}_{b}$ for the position vector of the sphere relative to the ellipsoid. Relative to their center of mass, we also have the positions

$$
\boldsymbol{r}_{e}=-v \boldsymbol{r}_{b}
$$

$$
\boldsymbol{r}_{s}=(1-v) \boldsymbol{r}_{b}
$$

where subscripts $s$ and $e$ refer to the sphere and the ellipsoid, respectively.

Taken from [1] for a general body in a sphere restricted binary system, the dynamics of the binary system in the general body-fixed frame is defined by

$$
\ddot{\boldsymbol{r}}_{b}+2 \boldsymbol{\Omega} \times \dot{\boldsymbol{r}}_{b}+\dot{\boldsymbol{\Omega}} \times \boldsymbol{r}_{b}+\boldsymbol{\Omega} \times\left(\boldsymbol{\Omega} \times r_{b}\right)=G\left(M_{1}+M_{2}\right) \frac{\partial \tilde{U}}{\partial \boldsymbol{r}_{b}}
$$




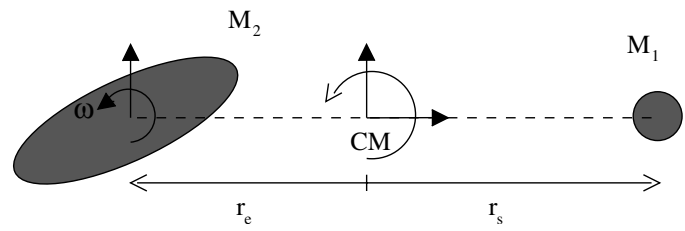

Fig. 1 Representation of the full two-body problem for an ellipsoidsphere system.

and the rotational dynamics of the general body are described in the general body-fixed frame by

$$
\boldsymbol{I} \cdot \dot{\boldsymbol{\Omega}}+\times \boldsymbol{I} \cdot \boldsymbol{\Omega}=-G M_{1} \boldsymbol{r}_{b} \times \frac{\partial \tilde{U}}{\partial \boldsymbol{r}_{b}}
$$

where $\boldsymbol{\Omega}$ is the angular velocity of the general body, $\boldsymbol{I}$ is its inertia matrix normalized by its mass and $\tilde{U}$ is the mutual potential, defined as

$$
\tilde{U}=\frac{1}{M_{2}} \int_{\beta_{2}} \frac{\mathrm{d} m_{2}(\boldsymbol{\rho})}{\left|\boldsymbol{r}_{\mathrm{b}}+\boldsymbol{\rho}\right|}
$$

The variable $\rho$ is the position vector of a mass element of the general body.

To simplify the analysis, the following normalizations are introduced. The maximum radius of the distributed body, denoted by $\alpha$, and the mean motion of the system at this radius

$$
n=\sqrt{\frac{G\left(M_{1}+M_{2}\right)}{\alpha^{3}}}
$$

are taken as length and time scales, respectively. The normalized position and angular velocity are then

$$
\boldsymbol{r}=\frac{\boldsymbol{r}_{b}}{\alpha} \quad \text { and } \quad \boldsymbol{\omega}=\frac{\boldsymbol{\Omega}}{n}
$$

and Eqs. (4) and (ㄷ) now become

$$
\begin{gathered}
\ddot{\boldsymbol{r}}+2 \boldsymbol{\omega} \times \dot{\boldsymbol{r}}+\dot{\boldsymbol{\omega}} \times \boldsymbol{r}+\boldsymbol{\omega} \times(\boldsymbol{\omega} \times \boldsymbol{r})=\frac{\partial U}{\partial \boldsymbol{r}} \\
\boldsymbol{I} \cdot \dot{\boldsymbol{\omega}}+\boldsymbol{\omega} \times \boldsymbol{I} \cdot \boldsymbol{\omega}=-v \boldsymbol{r} \times \frac{\partial U}{\partial \boldsymbol{r}}
\end{gathered}
$$

In this work, we model the general body as an ellipsoid. As discussed in [7], the general expression for an ellipsoid potential energy is written in terms of elliptic integrals. The mutual potential can be expressed as

$$
\begin{gathered}
U_{e}=\frac{3}{4} \int_{\lambda}^{\infty} \phi(\boldsymbol{r}, v) \frac{\mathrm{d} v}{\Delta(v)} \\
\phi(\boldsymbol{r}, v)=1-\frac{x^{2}}{v+1}-\frac{y^{2}}{v+\beta^{2}}-\frac{z^{2}}{v+\gamma^{2}} \\
\Delta(v)=\sqrt{(v+1)\left(v+\beta^{2}\right)\left(v+\gamma^{2}\right)}
\end{gathered}
$$

where $0<\gamma \leq \beta \leq 1$ and $\lambda$ satisfies $\phi(\boldsymbol{r}, \lambda)=0$. In this work, the $x$ axis is aligned with the longest axis of the ellipsoid, whereas the $z$ axis is along its shortest axis. The normalized principal moments of inertia are

$$
\begin{gathered}
I_{x x}=\frac{1}{5}\left(\beta^{2}+\gamma^{2}\right) \\
I_{y y}=\frac{1}{5}\left(1+\gamma^{2}\right)
\end{gathered}
$$

$$
I_{z z}=\frac{1}{5}\left(1+\beta^{2}\right)
$$

From Eqs. (7) and (8), we can solve for relative equilibria when all first and second derivatives are zero. With these same equations, it is then possible to show that there exists equilibria when one of the principal axes of the ellipsoid is pointed at the sphere, as the acceleration and position vectors are parallel. Similarly, we can show that the spin vector is perpendicular to the position vector, and it is parallel to one of its principal axes. These equilibria are independent of the principal axis the ellipsoid is rotating about; solutions exist along the $x, y$, and $z$ axis. Given a solution along a $q$ axis, which can be $x, y$, or $z$, the square of the spin rate is expressed as[3]

$$
\omega^{2}=\frac{3}{2} \int_{\lambda}^{\infty} \frac{\mathrm{d} v}{\left(\alpha_{q}^{2}+v\right) \Delta(v)}
$$

Note that the spin may be about one of the other two principal axes. For the present problem, $\alpha_{q}$ represents the radius along which the sphere is located. Note that here, $\lambda=q^{2}-\alpha_{q}^{2}$, in which $q$ is the distance between the primaries. In our analysis, we denote $q$ as $r$, with the ellipsoid having $\alpha_{q}=\alpha=1$.

The relative equilibria for an ellipsoid-sphere system and their stability have been mapped in [1] as a function of the mass ratio and distance between the bodies. This work studied two configurations in which the $\alpha$ or $\beta$ ellipsoid parameter is aligned with the sphere, referred to as the long-axis and short-axis configurations, respectively. We choose to consider the long-axis configuration, as it was shown to be the only energetically stable configuration for certain parameters. Hence, in Eq. (12), we look at the case in which $r$ is along the $x$ axis. Note that following a more general discussion of the F2BP in [2], the spectral and energetic stability of the ellipsoidsphere system relative equilibria were also studied for the case of a fixed value of angular momentum [8]; however, this topic is not addressed in the current paper.

\section{Restricted Full Three-Body Problem}

\section{A. Equations of Motion of a Particle}

Having a relative equilibrium state in the F2BP, we look at the dynamics of a particle in this gravitational field. Earlier studies looked at equilibrium solutions, especially the equilateral Lagrangian points and their stability [4]. By investigating the collinear points, zero-velocity curves and the Jacobi integral, one can give a general description of the constraints on a particle's dynamics.

We now consider a particle or a spacecraft in the gravitational field of the binary system. For the current problem, we assume relative equilibrium of the F2BP as defined by Eqs. (7) and (8). The equations of motion of a particle were originally defined in [3]. As shown in Fig. 2, we now let $\rho$ be the position of a particle relative to the center of mass of the system. The dynamics are expressed as

$$
\ddot{\boldsymbol{\rho}}+2 \boldsymbol{\Omega} \times \dot{\boldsymbol{\rho}}+\boldsymbol{\Omega} \times(\boldsymbol{\Omega} \times \boldsymbol{\rho})=G\left(M_{1}+M_{2}\right) \frac{\partial \tilde{U}_{12}}{\partial \boldsymbol{\rho}}
$$

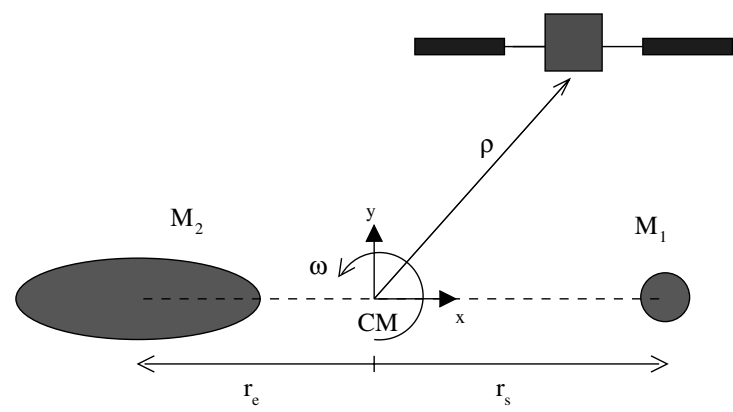

Fig. 2 The restricted full three-body problem. 
In normalized units, Eq. (13) becomes

$$
\ddot{\tilde{\rho}}+2 \omega \times \dot{\tilde{\rho}}+\omega \times(\omega \times \tilde{\rho})=\frac{\partial U_{12}}{\partial \tilde{\rho}}
$$

where

$$
\tilde{\rho}=\frac{\rho}{\alpha}
$$

is the normalized distance of the particle. Because we take a frame fixed to the ellipsoid, $U_{12}$ is a time-invariant potential energy expression, as the bodies are in mutual equilibrium. It is expressed as

$$
U_{12}=\frac{v}{|\tilde{\boldsymbol{\rho}}-(1-v) \boldsymbol{r}|}+(1-v) U_{e}(\tilde{\boldsymbol{\rho}}+v \boldsymbol{r})
$$

$U_{e}$ represents the normalized expression for the ellipsoid body, defined by Eqs. (9-11).

The free parameters of this system are the mass ratio, $v$, the distance between the two bodies, $r$, and the size parameters of the ellipsoid, $\beta$ and $\gamma$. Given these parameters, the spin rate $\omega$ is given by Eq. (12). The equations of motion can be restated in an $(x, y, z)$ coordinate system; that is, $\tilde{\boldsymbol{\rho}}=x \boldsymbol{i}+y \boldsymbol{j}+z \boldsymbol{k}$. The $x, y$, and $z$ components of Eq. (14) are written as follows:

$$
\begin{gathered}
\ddot{x}-2 \omega \dot{y}-\omega^{2} x=\frac{-v[x-(1-v) r]}{\left\{[x-(1-v) r]^{2}+y^{2}+z^{2}\right\}^{\frac{3}{2}}} \\
-(1-v)(x+v r) R_{j \alpha} \\
\ddot{y}+2 \omega \dot{x}-\omega^{2} y=\frac{-v y}{\left\{[x-(1-v) r]^{2}+y^{2}+z^{2}\right\}^{\frac{3}{2}}}-(1-v)(y) R_{j \beta} \\
\ddot{z}=\frac{-v z}{\left\{[x-(1-v) r]^{2}+y^{2}+z^{2}\right\}^{\frac{3}{2}}}-(1-v)(z) R_{j \gamma}
\end{gathered}
$$

The $R_{j}$ expressions are elliptic integrals taking into account the mass distribution of the ellipsoid. They are given in the Appendix.

\section{B. Equilibrium Solutions}

As for the R3BP, five equilibrium solutions exist when velocities and accelerations are set to zero in Eq. (14), and are solutions of the equation

$$
\boldsymbol{\omega} \times(\boldsymbol{\omega} \times \tilde{\boldsymbol{\rho}})=\frac{\partial U_{12}}{\partial \tilde{\boldsymbol{\rho}}}
$$

These five locations are shown qualitatively in Fig. 3 . The equilibrium solutions are then computed from

$$
\omega^{2} x=\frac{v[x-(1-v) r]}{\left\{[x-(1-v) r]^{2}+y^{2}+z^{2}\right\}^{\frac{3}{2}}}+(1-v)(x+v r) R_{j \alpha}
$$

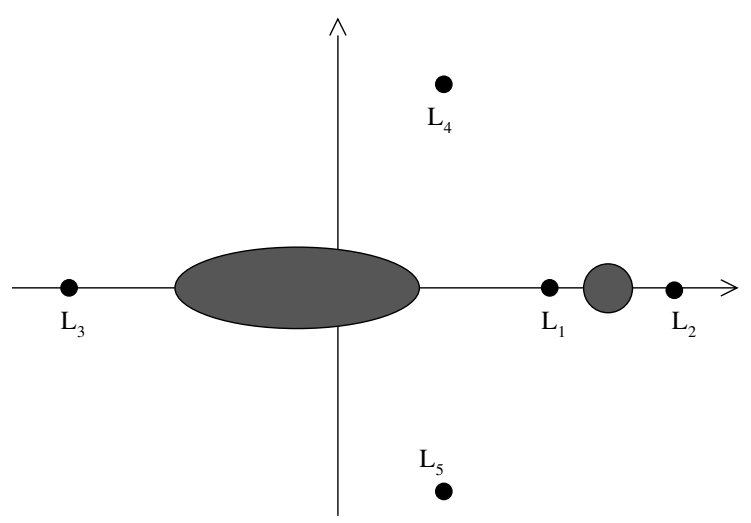

Fig. 3 The analog Lagrangian points.

$$
\omega^{2} y=\frac{v y}{\left\{[x-(1-v) r]^{2}+y^{2}+z^{2}\right\}^{\frac{3}{2}}}+(1-v) y R_{j \beta}
$$

where $\omega$ is given by Eq. (12).

These algebraic equations were solved numerically using MATLAB for varying free parameters. Note that $z=0$ in all cases. The Lagrangian points can also be defined using the Jacobi integral of the system. This topic is introduced in the next section.

\section{Spectral Stability}

In the R3BP, for all values of the free parameters, $L_{1}, L_{2}$, and $L_{3}$ are unstable, whereas $L_{4,5}$ may be stable. Looking at small deviations from the equilateral position, we can investigate their stability. In the R3BP, the stability criteria for $L_{4,5}$ is usually given by the Routh criteria and is only function of the mass ratio:

$$
v<\frac{1}{2}\left[1-\sqrt{\frac{23}{27}}\right]=0.0385 \ldots
$$

Because the mass distribution of the general body is now taken into account, the stability of some of the equilibrium solutions are expected to deviate from the R3BP. We introduce $x=\tilde{x}+\mathrm{d} x$ and $y=\tilde{y}+\mathrm{d} y$, and expand the potential energy expression. The following equations are derived:

$$
\begin{aligned}
& \ddot{\tilde{x}}-2 \omega \dot{\tilde{y}}-\omega^{2} \tilde{x}=\tilde{x}\left(U_{x x s}+U_{x x e}\right)+\tilde{y}\left(U_{x y s}+U_{x y e}\right) \\
& \ddot{\tilde{y}}+2 \omega \dot{\tilde{x}}-\omega^{2} \tilde{y}=\tilde{y}\left(U_{y y s}+U_{y y e}\right)+\tilde{x}\left(U_{y x s}+U_{y x e}\right)
\end{aligned}
$$

The second order partial derivatives for the ellipsoid potential were given in [1]. The characteristic equation for the system is found from

$$
\left|\begin{array}{cc}
\lambda^{2}-\omega^{2}-U_{x x} & -2 \omega \lambda-U_{x y} \\
2 \omega \lambda-U_{x y} & \lambda^{2}-\omega^{2}-U_{y y}
\end{array}\right|=0
$$

where $U_{x x}=U_{x x s}+U_{x x e}, U_{y y}=U_{y y s}+U_{y y e}$, and $U_{x y}=U_{x y s}+$ $U_{\text {xye }}$.

Expanding the determinant, the characteristic equation is found to be of the form

$$
\lambda^{4}+A \lambda^{2}+B=0
$$

where

$$
A=2 \omega^{2}-U_{x x}-U_{y y}
$$

and

$$
B=\omega^{4}+\omega^{2}\left(U_{x x}+U_{y y}\right)+U_{x x} U_{y y}-U_{x y}^{2}
$$

For the system to be linearly stable, the following conditions must be satisfied:

$$
\begin{gathered}
A>0 \\
B>0 \\
A^{2}-4 B>0
\end{gathered}
$$

As in the R3BP, the collinear Lagrangian points $L_{1,2,3}$ are unstable. The equilateral Lagrangian points were studied in [4]. For these, the stability was found to be decreased from the known R3BP (also see Fig. 7). We give a more detailed description of $L_{4,5}$ with the discussion on energy constraints in the next section. 


\section{Energy Constraints}

\section{A. Jacobi Integral}

With the two bodies being in relative equilibrium, this system allows for one integral of motion, the Jacobi integral. For a spacecraft navigating in this system, computing its Jacobi integral value is important, as it indicates the regions in which it can move and provides necessary conditions for when it may escape the system. To compute the integral, let us rewrite Eqs. (16) and (17) in the following form:

$$
\begin{gathered}
\ddot{x}-2 \omega \dot{y}=\frac{\partial V}{\partial x} \\
\ddot{y}+2 \omega \dot{x}=\frac{\partial V}{\partial y} \\
\ddot{z}=\frac{\partial V}{\partial z}
\end{gathered}
$$

where

$$
V=-\frac{v}{\left|\rho-\boldsymbol{r}_{s}\right|}-U_{e}\left(\boldsymbol{\rho}-\boldsymbol{r}_{e}\right)+\frac{1}{2} \omega^{2}\left(x^{2}+y^{2}\right)
$$

with

$$
r_{s}=\sqrt{\left\{[x-(1-v) r]^{2}+y^{2}\right\}}
$$

and

$$
U_{e}=\frac{3}{2}(1-v) R_{j 0}-\frac{1}{2}(1-v)\left[(x+v r)^{2} R_{j x}+y^{2} R_{j y}\right]
$$

To derive the Jacobi integral, we multiply each of the two previous equations by $\dot{x}$ and $\dot{y}$, respectively, and add them up to find

$$
\ddot{x} \dot{x}+\ddot{y} \dot{y}+\ddot{z} \dot{z}-\omega^{2}(\dot{x} x+\dot{y} y)=\frac{\partial V}{\partial x} \dot{x}+\frac{\partial V}{\partial y} \dot{y}+\frac{\partial V}{\partial z} \dot{z}
$$

We substitute

$$
\ddot{x}=\frac{\mathrm{d} \dot{x}}{\mathrm{~d} t}=\frac{\mathrm{d} \dot{x}}{\mathrm{~d} x} \frac{\mathrm{d} x}{\mathrm{~d} t}
$$

and integrate with respect to time. Equation (36) becomes

$$
C=\frac{1}{2}\left(\dot{x}^{2}+\dot{y}^{2}+\dot{z}^{2}\right)-V
$$

where $C$ is called the Jacobi constant, the integral value of this system, or, more generally,

$$
C=\frac{1}{2} v_{R}^{2}-V
$$

where $v_{R}$ is the speed of a particle or a spacecraft relative to the rotating frame.

Relating the relative velocity to inertial velocity, the Jacobi constant is then written as

$$
C=\frac{1}{2} v_{\mathrm{I}}^{2}-(\boldsymbol{\omega} \times \boldsymbol{r}) \cdot \boldsymbol{v}_{\mathrm{I}}-U_{12}
$$

where we have

$$
\boldsymbol{v}_{\mathrm{I}}=\boldsymbol{v}_{R}+\omega \times \boldsymbol{r}
$$

And we substitute for $v_{R}^{2}$ using

$$
\boldsymbol{v}_{R} \cdot \boldsymbol{v}_{R}=v_{\mathrm{I}}^{2}-2(\boldsymbol{\omega} \times \boldsymbol{r}) \cdot \boldsymbol{v}_{\mathrm{I}}+\omega^{2}\left(x^{2}+y^{2}\right)
$$

In either form, given values of the Jacobi constants, there exist constraints on the motion of a particle. We start with a study of the zero-velocity curves.

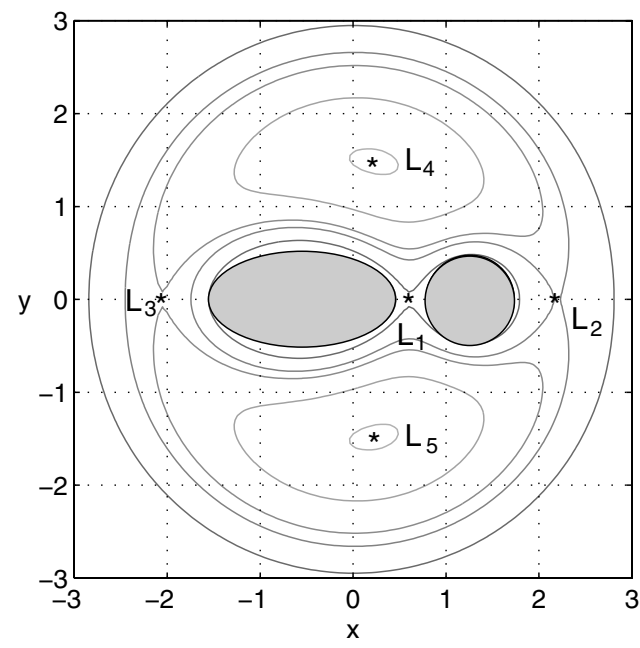

Fig. 4 Zero-velocity curves in the $x-y$ coordinate frame for an ellipsoidsphere system with distance between the bodies of $r=1.8$; ellipsoid parameters $\alpha=1, \beta=0.5$, and $\gamma=0.5$; and mass ratio of $v=0.3$. The small darker circle and ellipse represent the bodies themselves.

\section{B. Zero-Velocity Curves}

In Eq. (38), the solutions of $C=-V$ delineate between the allowable and nonallowable motion of a spacecraft in this gravitational field, or the zero-velocity curves. If $C \ll-1$, then $V \gg 1$, meaning $x$ and $y$ can be either large or very small to satisfy the relation. This restricts the motion of the spacecraft to be either far away from the bodies or very close to them. In Fig. 4 , these two cases correspond to the regions exterior to the large circular line around the bodies, referred to as the outer region, and the small circle near the bodies. Note that the small darker circle and ellipse represent the two components of the binary system. In the outer region, a spacecraft can escape from the system, whereas in the interior region it cannot. Note that with mass distributions, it becomes necessary to account for impact on the surface.

Increasing the value of $C$ allows one to define the three collinear Lagrangian points. First, the two zero-velocity curves close to each body will meet at one point on the $x$ axis between the bodies. This defines the $L_{1}$ Lagrangian point with its Jacobi constant, $C_{1}$, associated with it. The ellipsoid, having a finite size, adds one interesting constraint on the location of the $L_{1}$ point. The limiting case is when $L_{1}$ sits on the ellipsoid, facing the sphere. Figure 5 provides a closer view of this situation. In this case, we have

$$
x_{L_{1}}=1-v r
$$

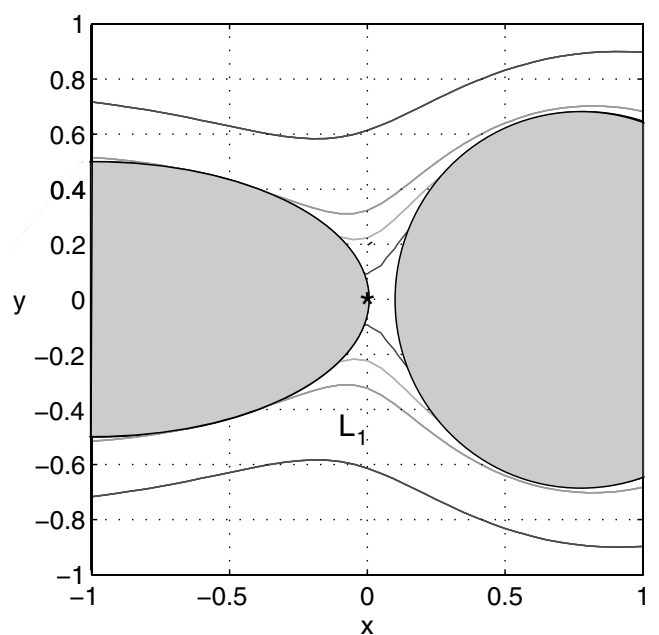

Fig. 5 Zero-velocity curves for an ellipsoid-sphere system in which $L_{1}$ is sitting on the ellipsoid surface, facing the sphere. The parameters are $r=1.8, v=0.5586, \beta=\gamma=0.5$. The black lines represent the bodies themselves. 


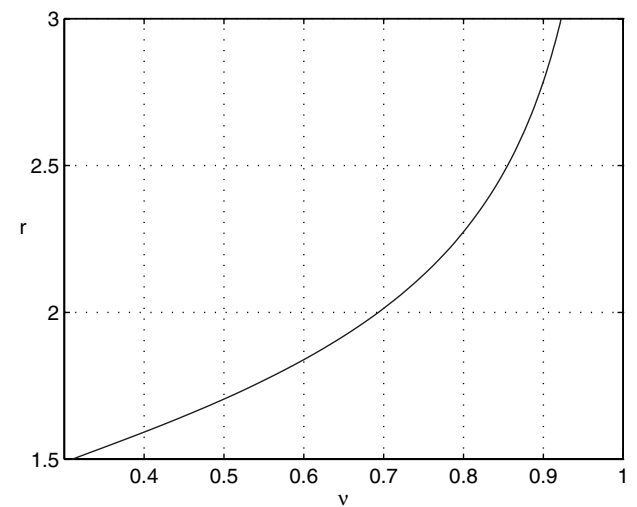

Fig. 6 Values of the distance between the bodies, $r$, as a function of the mass ratio $v$ to have the $L_{1}$ Lagrangian point sitting on the ellipsoid body, facing the sphere. The ellipsoid parameters are $[\alpha: \beta: \gamma]=[1: 0.5: 0.5]$.

We can then substitute $x_{L_{1}}$ with $y=z=0$ into Eq. (20) and solve for the mass ratio as a function of the distance between the bodies, $r$ :

$$
v=\frac{\omega^{2}-\frac{2}{3} R_{j x}}{\left(\omega^{2} r-\frac{1}{(r-1)^{2}}-\frac{2}{3} R_{j x}\right)}
$$

Hence, given a value of the distance between the bodies, Eq. (43) gives the corresponding mass ratio to have $L_{1}$ sitting on the edge of the ellipsoid, facing the sphere. Depending on the system parameters, $L_{1}$ can be either inside or outside of the ellipsoid body. Figure $\underline{6}$ shows the mass ratio as a function of the distance between the primaries satisfying Eq. (43). The upper region of the curve defines the parameters for which $\bar{L}_{1}$ is outside of the ellipsoid; the lower region represents cases of $L_{1}$ being inside the ellipsoid. A variety of trajectories can be computed for motion in the vicinity of $L_{1}$, and they are addressed in the following section.

Then, increasing $C$ again allows the inner region to meet with the outer region of allowable motion, on one side of the binary system and then on the other side, defining the $L_{2}$ and $L_{3}$ Lagrangian points. The point at which the two regions meet first depends on the free parameters of the system. The two points will appear at the same time for a mass ratio of about $v=0.5$, depending on the ellipsoid parameters. This meeting point happens for slightly smaller mass ratios in the case of a pronounced ellipsoid. Note that we keep the same notation on the Lagrangian points throughout the text, independently of the point, $L_{2}$ or $L_{3}$ appearing first. The case of interest is for binary systems with large mass ratios (i.e., small ellipsoid body), as such systems have been found to be stable and to exist in nature [1,5]. In such cases, from the convention on Lagrangian points introduced in Fig. $3, L_{3}$ appears first on the exterior side of the ellipsoid.

Finally, as for the R3BP, $L_{4}$ and $L_{5}$ are then defined as being the two points forming in the vicinity of the equilateral triangle points in the R3BP. Note that these two Lagrangian points are mirrors of each other about the $x$ axis. In general, they are stable only for very small or very large mass ratios. The location and stability of these points were studied in [4]. Figures $7 \mathrm{a}$ and $7 \mathrm{~b}$ show results for a long-axis configuration with the distance, $r=2$, between the bodies. On Fig. $7 \mathrm{a}$, the mass ratio $v$ varies from 0 to 1 horizontally from left to right, and $\gamma=\beta$ varies from 0 to 1 vertically from bottom to top. Starred points are stable, whereas dotted ones are unstable. On Fig. $7 \mathrm{~b}$, each line corresponds to different values of $\gamma / \beta$ and equal $0.25, \overline{0} .5,0.75$, and 1.0. Stable regions lie above the lines in the upper figure and below the lines in the lower figure. The horizontal dotted line corresponds to the Routh criterion. We see that the stability region is reduced from the $\mathrm{R} 3 \mathrm{BP}$, although exceptions exist for small mass ratios.

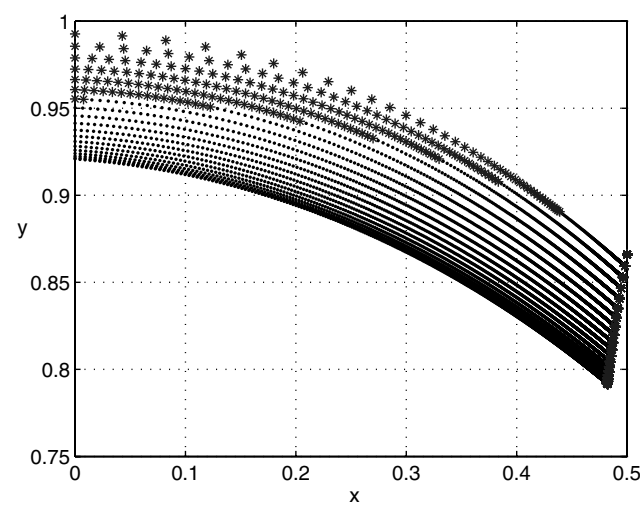

a)
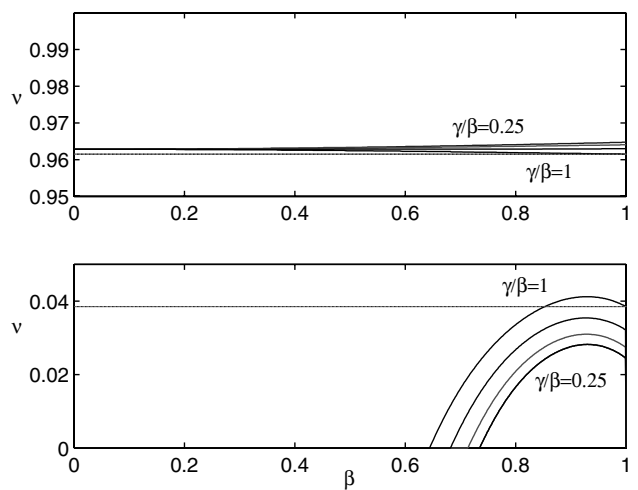

b)

Fig. 7 a) Locations of the analog equilibrium points for $r=2$ in the $x-y$ coordinate space for the long-axis configuration. The mass ratio $v$ varies from 0 to 1 horizontally from left to right and $\gamma=\beta$ varies from 0 to 1 vertically from bottom to top. Starred points are stable, whereas dotted ones are unstable. b) Stability regions of the long-axis configuration for $r=2$ as a function of $\beta$ and $v$. The lines correspond to different values of $\gamma / \beta$ and equal $0.25,0.5,0.75$, and 1.0. Stable regions lie above the lines in the upper figure and below the lines in the lower figure. The horizontal dotted line corresponds to the Routh criterion.

\section{Surface Motion in an Ellipsoid-Sphere Binary System}

\section{A. Trajectory Investigations Using Linearization Near $L_{1}$}

An eventual mission to a binary system would want to carry out surface motion for scientific investigations or for sample return objectives. Other than surface and gravity constraints, situations such as spinning of the bodies need to be taken into account. In terms of mission design, for a small ellipsoid case, it would be interesting to have a vehicle approaching a binary system from the small ellipsoid body, through $L_{3}$, as the primary is often spinning more rapidly than the orbit rate [5]. Given a value of the Jacobi integral, the spacecraft would have enough energy to travel close to the system, visiting both bodies, without escaping through the $L_{3}$ region. Hence, it is important to look into possible missions scenarios and to characterize the surface conditions and requirements for possible transit trajectories between the bodies.

For certain parameters of the system, $L_{1}$ is on the outside of the ellipsoid, providing a channel for possible transit trajectories. However, because of the instability of $L_{1}$, only certain conditions on the position and velocity of a spacecraft can lead to transit. Moreover, certain velocity limits are required to prevent a vehicle from escaping the system.

Different trajectories such as transit and nontransit trajectories between the bodies can be analyzed from linearizing near $L_{1}$ and computing its manifolds. In the following, we apply the methodology of Conley [9] to our problem. This is possible by investigating the eigenvalues and eigenvectors of the state transition matrix evaluated about $L_{1}$. The $L_{1}$ Equilibrium 
point has one pair of real and one pair of imaginary conjugate eigenvalues. The corresponding eigenvectors, one pair of hyperbolic manifolds and one center manifold, respectively, make $L_{1}$ unstable.

To find the manifolds at $L_{1}$, we first compute the eigenvalues of the linearized dynamics. For a system of the form $\dot{x}=F(x, t)$, the linearized equations are computed using the first derivative of $F(x, t), \partial F / \partial x$. For the dynamics defined by Eqs. (16) and (17), we express

$$
\frac{\partial F}{\partial x}=\left[\begin{array}{cccc}
0 & 0 & 1 & 0 \\
0 & 0 & 0 & 1 \\
\omega^{2}+\left(U_{x x s}+U_{x x e}\right) & \left(U_{x y s}+U_{x y e}\right) & 0 & 2 \omega \\
\left(U_{x y s}+U_{x y e}\right) & \omega^{2}+\left(U_{y y s}+U_{y y e}\right) & -2 \omega & 0
\end{array}\right]
$$

Note that the second-order derivatives for the sphere and ellipsoid potential are given in [4].

Because of the nature of $L_{1}$, we can write the eigenvalues as $\pm \lambda_{1}$ and $\pm \lambda_{2}$, in which $\lambda_{1}$ is real and $\lambda_{2}$ is imaginary. The associated eigenvectors are $\boldsymbol{\mu}_{1}^{ \pm}$and

$$
\boldsymbol{\mu}_{2}=\boldsymbol{\mu}_{2}^{\mathrm{Re}}+i \boldsymbol{\mu}_{2}^{\mathrm{Im}}
$$

The solution for the particle dynamics can be written as a superposition of the eigenvectors.

$$
\boldsymbol{q}=\alpha^{+} \boldsymbol{\mu}_{1}^{+} e^{\left(\lambda_{1} t\right)}+\alpha^{-} \boldsymbol{\mu}_{1}^{-} e^{\left(-\lambda_{1} t\right)}+2 \operatorname{Re}\left(\beta \boldsymbol{\mu}_{2} e^{\left(i \lambda_{2} t\right)}\right)
$$

where $\alpha^{+}, \alpha^{-}$, and $\beta$ are constants.

Now, let us substitute

$$
\beta=\beta^{\mathrm{Re}}+i \beta^{\mathrm{Im}}
$$

and

$$
e^{\left(i \lambda_{2} t\right)}=\cos \left(\lambda_{2} t\right)+i \sin \left(\lambda_{2} t\right)
$$

Equation (45) then becomes

$$
\begin{aligned}
\boldsymbol{q} & =\alpha^{+} \boldsymbol{\mu}_{1}^{+} e^{\left(\lambda_{1} t\right)}+\alpha^{-} \boldsymbol{\mu}_{1}^{-} e^{\left(-\lambda_{1} t\right)}+2 \beta^{\mathrm{Re}}\left[\boldsymbol{\mu}_{2}^{\mathrm{Re}} \cos \left(\lambda_{2} t\right)\right. \\
& \left.-\boldsymbol{\mu}_{2}^{\mathrm{Im}} \sin \left(\lambda_{2} t\right)\right]-2 \beta^{\mathrm{Im}}\left[\boldsymbol{\mu}_{2}^{\mathrm{Re}} \sin \left(\lambda_{2} t\right)+\boldsymbol{\mu}_{2}^{\mathrm{Im}} \cos \left(\lambda_{2} t\right)\right]
\end{aligned}
$$

With $t=0$ in Eq. (48), we have

$$
\boldsymbol{q}=\left[\boldsymbol{\mu}_{1}^{+}, \boldsymbol{\mu}_{1}^{-}, \mathbf{2} \boldsymbol{\mu}_{2}^{\mathrm{Re}},-\mathbf{2} \boldsymbol{\mu}_{2}^{\mathrm{Im}}\right]\left[\begin{array}{c}
\alpha^{+} \\
\alpha^{-} \\
\beta^{\mathrm{Re}} \\
\beta^{\mathrm{Im}}
\end{array}\right]
$$

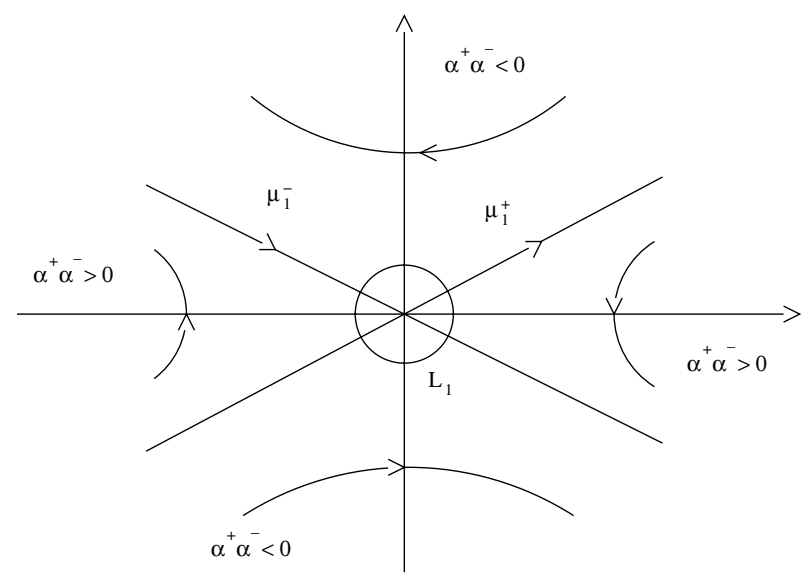

Fig. 8 Transit and nontransit trajectory regions at $L_{1}$.

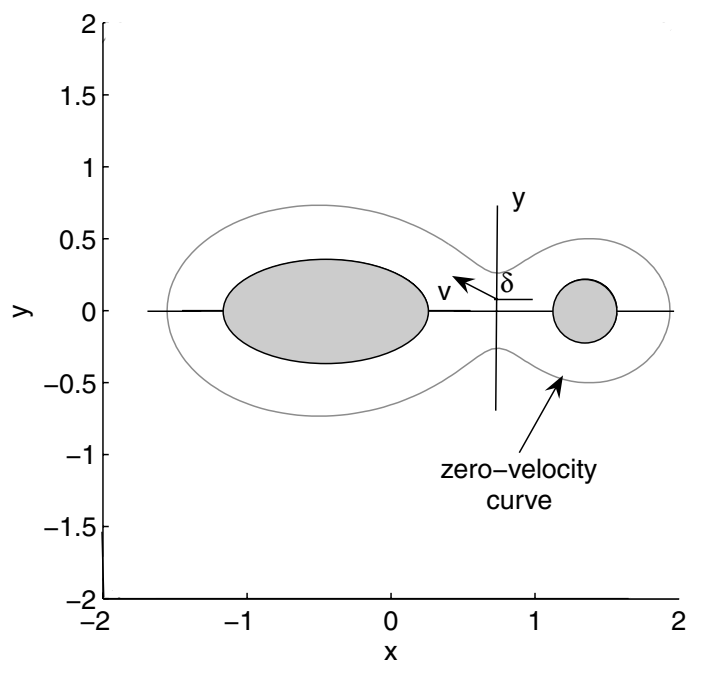

Fig. 9 Geometry of the transit/nontransit trajectories investigation at $L_{1}$ when it is outside of the ellipsoid body.

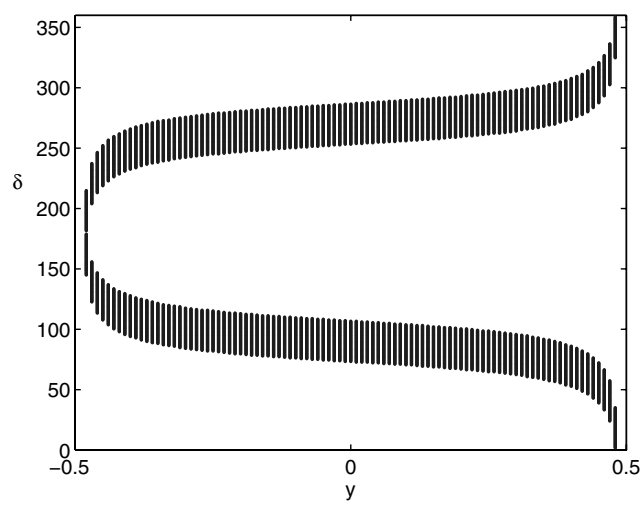

Fig. 10 Results on transit and nontransit trajectories at $L_{1}$ for $r=2$, $\beta=\gamma=0.5$, and $v=0.3$ across $y=[-0.5: 0.5]$. The dark region indicates nontransit trajectories.

Writing Eq. (49) in the form $\boldsymbol{q}=[\boldsymbol{\mu}] \boldsymbol{\alpha}_{\mu}$, we can find the components of $\boldsymbol{\alpha}_{\mu}$ from

$$
\boldsymbol{\alpha}_{\mu}=[\boldsymbol{\mu}]^{-1} \boldsymbol{q}
$$

Having the constants $\alpha^{+}, \alpha^{-}, \beta^{\mathrm{Re}}$ and $\beta^{\mathrm{Im}}$, we can investigate trajectories of a particle near the $L_{1}$ Lagrangian point from its linear dynamics. Depending on the value of $\alpha^{+}$and $\alpha^{-}$, the system will excite different manifolds, leading to different types of trajectories. As shown on Fig. 8, we can summarize these cases as being transit trajectories, nontransit trajectories and asymptotic trajectories, respectively:

$$
\begin{aligned}
& \alpha^{+} \alpha^{-}<0 \\
& \alpha^{+} \alpha^{-}>0 \\
& \alpha^{+} \alpha^{-}=0
\end{aligned}
$$

To find regions allowing transit and nontransit trajectories, we computed the $\alpha$ constants along the $y$ axis of the $L_{1}$ point with varying the direction of the velocity vector. The geometry is sketched in Fig. 9. Results on the trajectories are shown in Fig. 10. The dark and white region represents nontransit and transit trajectories, respectively, for a system with $r=2, \beta=\gamma=0.5$, and $\nu=0.3$ for an interval on the $y$ axis of $[-0.5,0.5]$. 


\section{B. Surface Conditions Leading to Transit and Nontransit Trajectories}

We can integrate the methodology from previous sections to design a possible mission leading to surface exploration and transit between the bodies. As the gravity of an asteroid is low, we are interested in looking at surface motion from a "hopper" point of view; a vehicle would most likely bounce back from hitting the surface and loose traction. Hence, wheeled vehicles might be difficult to control and keep track of. In addition, a hopper would be able to investigate a larger area in quicker time and might be easier to control.

It is common to find binary systems made of a small ellipsoid and a large sphere having a faster spin rate than the mutual binary orbit rate. In this case, as defined in Fig. 3, its Lagrangian point $L_{3}$ is the first to open after $L_{1}$. Hence, considering a locked configuration of the bodies and a spinning primary, the spacecraft approaching the binary system through $L_{3}$ is the best approach to take. This also limits the probability of escaping from the system as the spacecraft could only exit from the same entrance region.

After being inserted close to the binary system, a spacecraft could place a vehicle on the surface for further exploration of both bodies. A hopper would easily move on the surface, investigating the small ellipsoid body and taking measurements along the way. The micro/ nano experimental robot vehicle for asteroid (MINERVA) robot of the Hayabusa (MUSES-C) mission provides a good example of such application [10]. Eventually, the vehicle would hop to the side facing the sphere and then travel across the $L_{1}$ region using a simple jump to investigate the massive spinning spheroidal body. For transfer between the bodies, we use the algorithm defined in Sec. V.A that relates possible transfer trajectories through $L_{1}$ and surface conditions.

Knowing the conditions giving transit and nontransit trajectories from linear investigation at $L_{1}$, we can integrate the system backward and forward in time to find initial and final conditions of the particle dynamics on the surface of either body. From the results on transit and nontransit trajectories shown on Fig. 10, we investigated conditions for transit trajectories. Figure $\overline{11}$ shows a typical nontransit trajectory for parameters $r=2, \beta=\bar{\gamma}=0.5$, and $v=0.3$ and two transit trajectories in the center and outer regions of the surface of the bodies. The small arrows show the locus of transit trajectories surface conditions; the region facing the bodies gives more transfer options, whereas the conditions need to be more precise for the outer regions on the surface.

We investigated different conditions of transit and nontransit trajectories crossing the $L_{1}$ region. The point A on Fig. 12a represents a fixed value on the $y$ axis at $L_{1}$ through which trajectories are crossing with varying directions $\delta$ defined in Fig. 9 .

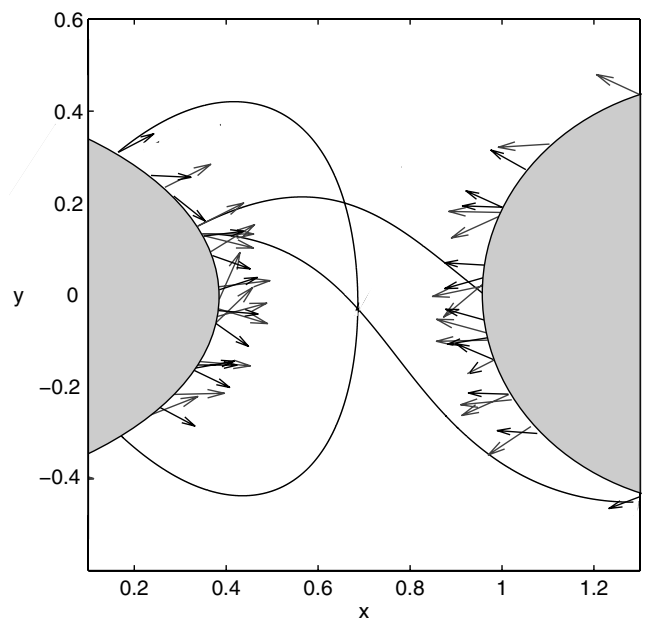

Fig. 11 Transit and nontransit trajectories for a binary system with $r=2, v=0.3$, and ellipsoid parameters $\beta=\gamma=0.5$. The arrows are initial and final conditions on the surface of the bodies leading to transit trajectories.

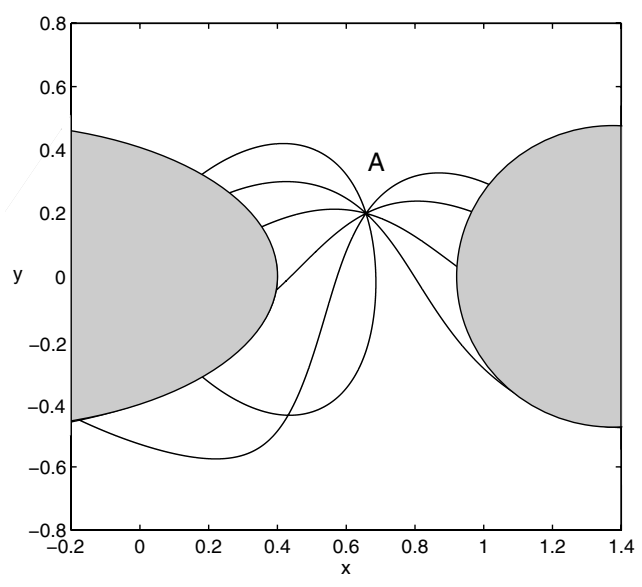

a)

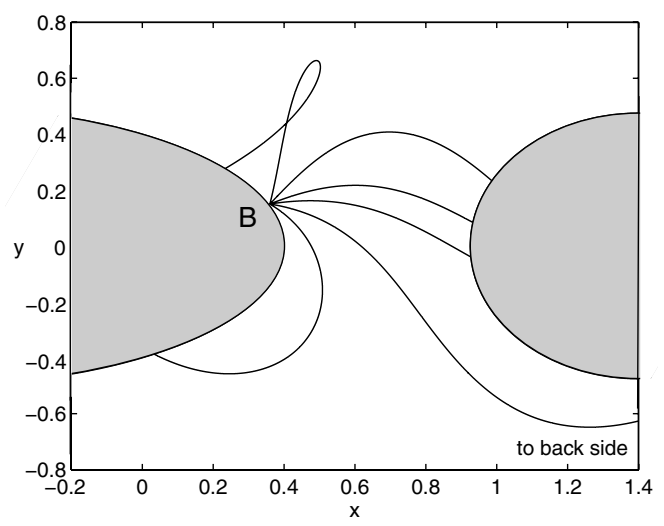

b)

Fig. 12 Transit and nontransit trajectories for a binary system with $r=2, v=0.3$, and ellipsoid parameters $\beta=\gamma=0.5$. a) Point A represents trajectories crossing $L_{1}$ at fixed value of $y, y=0.1$, with varying direction $\delta$. b) Point $B$ indicates different initial directions of launching velocities for a spacecraft leaving the surface of the ellipsoid.

We see that the corresponding surface conditions and nature of the trajectories can be very different while attempting to cross the $L_{1}$ region. Figure $12 \mathrm{~b}$ shows different initial velocity vectors for a vehicle leaving from the surface of the ellipsoid at point $B$. In this case, the direction of the launching velocity at the surface was varied although the initial surface location and the velocity magnitude were fixed. It can be seen that small differences in angle could lead to transfer or not. We also note that some initial conditions may lead to transfer to the back side of the spherical body.

\section{Bounds on Transfer Velocity}

Because we want to keep the spacecraft close to the bodies, we need to monitor the velocity involved in transferring from the ellipsoid to the sphere. Getting to an orbit encircling the binary system would make a spacecraft more susceptible to escape from the system. Given a Jacobi integral constant, we can find conditions on the velocity to make sure a spacecraft would not be able to escape from the system. Using these conditions, we can then perform the necessary maneuvers to satisfy the surface conditions leading to transit without possibilities of escaping.

Knowing that a spacecraft could have access to the outer region of the binary system by leaving through the $L_{2}$ or $L_{3}$ region, we can compute upper bounds on the transit velocity from their Jacobi integral values, $C_{2}$ and $C_{3}$, respectively. For the spacecraft being close to the back side of the ellipsoid, an energy value larger than $C_{3}$ would be a good upper bound, as $L_{3}$ opens first. If the spacecraft makes a transfer to the spinning sphere, an energy value larger than $C_{2}$ might be more suitable. 
Hence, in the design of a mission approaching the ellipsoid and transferring to the sphere, we use a value of energy larger than $C_{2}$ corresponding to an orbit encircling the binary as an upper bound on the spacecraft velocity. We refer to it as the outer region with $C_{\text {outer }}$ being the corresponding Jacobi value. Given a value of $C_{\text {outer }}$, we use Eq. (38) to obtain the corresponding velocity at the specific location on a body surface, and set it as $\boldsymbol{v}_{\max }$. This gives a bound on the velocity for the spacecraft to stay in orbit close to the binary system. The velocity correction for the spacecraft leaving the surface of the ellipsoid with a velocity $\boldsymbol{v}_{\text {sfc }}$ can be found from

$$
\Delta \boldsymbol{v}=\boldsymbol{v}_{\max }-\boldsymbol{v}_{\mathrm{sfc}}
$$

In the case of a spinning spherical primary with a spin rate given by $\boldsymbol{\Omega}_{\alpha}$, a vehicle on a spherical surface of radius $r_{\text {sfc }}$ would have a tangential velocity component given by

$$
\boldsymbol{v}_{T}=\left(\boldsymbol{\Omega}-\boldsymbol{\Omega}_{\mu}\right) \boldsymbol{r}_{\mathrm{sfc}}
$$

Note that the sphere spin $\boldsymbol{\Omega}_{\alpha}$ is usually faster than the binary orbit rate $\boldsymbol{\Omega}$ and that the Jacobi integral is not constant anymore. The transit velocity on the surface of the sphere is then

$$
\boldsymbol{v}_{\text {transit }}=\boldsymbol{v}_{T}+\boldsymbol{v}_{\mathrm{sfc}}
$$

From the results on the surface conditions given by Fig. 11, a vehicle would only need to hop in the right direction to bring the total launch velocity to the required magnitude and direction for transit. We want to make sure that the energy of the spacecraft stays low enough when it hits the spinning sphere. Hence, arriving at the sphere we can monitor the velocity using Eq. (54) and make the necessary maneuvers.

\section{Application to Binary System 1999 KW4}

We now consider the binary system 1999 KW4 as a case study [5]. We note that detailed models of both bodies are available, but they can be well approximated by a sphere for the primary and an ellipsoid for the secondary. The two bodies are referred to as $\alpha$ and $\beta$ for the sphere and ellipsoid, respectively, and the system has the following parameters. The distance between the bodies is $r_{b}=2.54 \mathrm{~km}$, the total mass of binary system is $M_{1}+M_{2}=2.472 \times 10^{12} \mathrm{~kg}$, with a mass ratio of $v=0.9457$, an orbit period $\Omega$ of $17.458 \mathrm{~h}$, and ellipsoid shape parameters

$$
(2 \alpha, 2 \beta, 2 \gamma)=(0.57: 0.455: 0.343) \mathrm{km}
$$

From these parameters, $r_{e}=-2.402 \mathrm{~km}$ and $r_{s}=0.138 \mathrm{~km}$. The spin rate of $\alpha$ is $2.8 \mathrm{~h}$, or

$$
\Omega_{\alpha}=6.23 \times 10^{-4} \mathrm{rad} / \mathrm{s}
$$

Also, $\beta$ is locked in a gravity gradient orbit with $\Omega_{\beta}=17.458 \mathrm{~h}$.

To work with nondimensional units, we set the scaling length and time as $\alpha=0.57 / 2$, and

$$
n=\sqrt{\left(\frac{G\left(M_{1}+M_{2}\right)}{\alpha^{3}}\right)}=2.67 \times 10^{-3} \mathrm{rad} / \mathrm{s}
$$

The nondimensional distance between the bodies becomes $r=8.9123$, and we solve for the rotation rate of the system using Eq. (12), $\omega=0.0377$. The nondimensional spin rate of the primary is then $\overline{\omega_{\alpha}}=0.233$.

\section{A. Equilibrium Points, Stability and Jacobi Constant}

To find the collinear equilibrium solutions, we solve Eq. (20) with $y=0$ and $z=0$. In nondimensional units, and following the convention on the Lagrangian points notations, we obtain, $L_{1}$ at [ $-6.2363 ; 0 ; 0], L_{2}$ at $[9.1004 ; 0 ; 0]$, and $L_{3}$ at $[-11.0158 ; 0 ; 0]$. The results indicate that $L_{1}$ is outside of the ellipsoid. For the equilateral

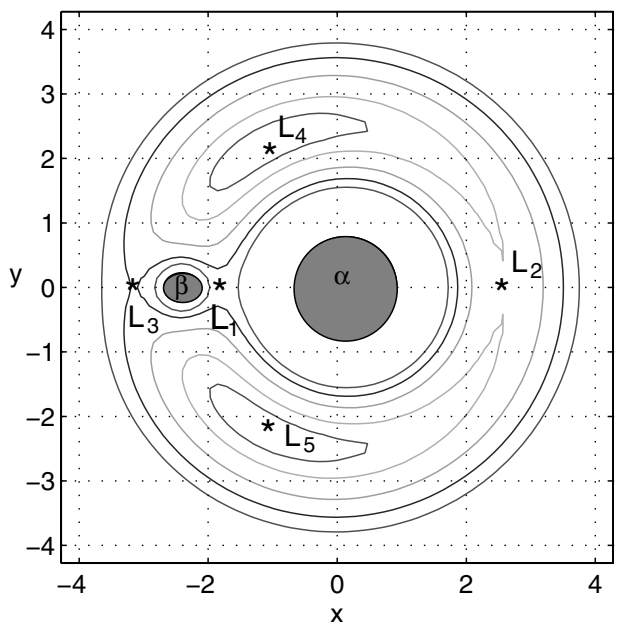

Fig. 13 Zero-velocity curve plot for $1999 \mathrm{KW} 4$ with $r=2.54 \mathrm{~km}$. The circle and ellipse around $\alpha$ and $\beta$ represent the bodies.

solutions, we find $L_{4,5}$ at $[-3.9713 ; \pm 7.7035 ; 0]$. In dimensional units, these give $L_{1}$ at $[-1.7773 ; 0 ; 0] \mathrm{km}, L_{2}$ at $[2.5936 ; 0 ; 0] \mathrm{km}, L_{3}$ at $[-3.1395 ; 0 ; 0] \mathrm{km}$, and $L_{4,5}$ at $[-1.1318 ; \pm 2.1955 ; 0] \mathrm{km}$.

Looking at the stability conditions given by Eq. (31), as expected, the collinear points are unstable. For the current mass ratio, $L_{4,5}$ are also unstable.

The corresponding Jacobi integrals, in nondimensional values, are $C_{1}=-0.19365, \quad C_{2}=-0.1716, \quad C_{3}=-0.18965$, and $C_{4,5}=$ -0.16565 . In dimensional units, we find $C_{1}=-1.1208 \times$ $10^{-7} \mathrm{~km}^{2} / \mathrm{s}^{2}, \quad C_{2}=-9.9323 \times 10^{-8} \mathrm{~km}^{2} / \mathrm{s}^{2}, \quad C_{3}=-1.0975 \times$ $10^{-7} \mathrm{~km}^{2} / \mathrm{s}^{2}$, and $C_{4}=-9.5883 \times 10^{-8} \mathrm{~km}^{2} / \mathrm{s}^{2}$. Figure 13 shows the zero-velocity curve plot for $1999 \mathrm{KW} 4$.

For a circular orbit about the sphere, say at $0.786 \mathrm{~km}$ radius, the velocity in the $\alpha$ frame is computed from

$$
v=\sqrt{\left(\frac{\mu}{r_{c}}\right)}=4.455 \times 10^{-4} \mathrm{~km} / \mathrm{s}
$$

where

$$
\mu=G M_{s}=1.5599 \times 10^{-7} \mathrm{~km}^{3} / \mathrm{s}^{2}
$$

Note that, from the spin itself, a particle would have a velocity of

$$
v_{\alpha}=\omega_{\alpha} r_{c}=4.897 \times 10^{-4} \mathrm{~km} / \mathrm{s}
$$

meaning particles are already close to being in orbit around $\alpha$. If we assume the spacecraft to be on the $x$ axis, the Jacobi value is calculated using Eq. (38).

If the spacecraft is located on the $x$ axis towards the system center of mass, it is at $-(0.786-0.138) \mathrm{km}$ in this coordinate system. Its associated Jacobi value is

$$
C_{x 1}=-2.0362 \times 10^{-7} \mathrm{~km}^{2} / \mathrm{s}^{2}
$$

If it is located on the other side of the sphere, it is at $(0.786+0.138) \mathrm{km}$, with

$$
C_{x 2}=-2.0114 \times 10^{-7} \mathrm{~km}^{2} / \mathrm{s}^{2}
$$

Because these values of the Jacobi are smaller than all the three values of the collinear points, these points have not opened up yet to the spacecraft on the surface of $\alpha$. Hence, there is no escape possible from orbiting close to the surface of $\alpha$.

From the zero-velocity curves shown on Fig. 13, a spacecraft or particle could be in the outer region if its Jacobi integral is higher than $C_{\text {outer }}=-0.19$ or

$$
C_{\text {outer }}=-1.0996 \times 10^{-7} \mathrm{~km}^{2} / \mathrm{s}^{2}
$$

This value corresponds to the large circular path around the binary 


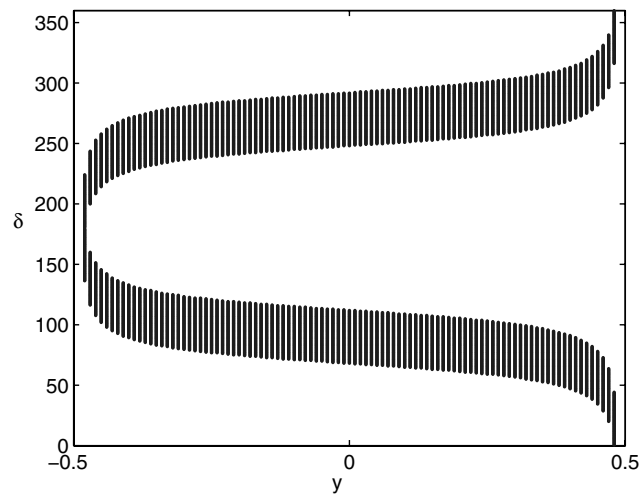

Fig. 14 Results on nontransit trajectories at $L_{1}$ for the binary 1999 KW4: $r=2.54 \mathrm{~km}, \quad \beta=0.8, \quad \gamma=0.6, \quad$ and $v=0.9457$ across $y=[-0.5: 0.5]$.

system. Inverting Eq. (38), for a spacecraft on the surface of $\alpha$, between the bodies, it would need to have a velocity of at least

$$
v_{\max \alpha}=8.695 \times 10^{-4} \mathrm{~km} / \mathrm{s}
$$

to have access to the outer region. On the surface of $\beta$, its velocity would only need to be

$$
v_{\max \beta}=1.95 \times 10^{-4} \mathrm{~km} / \mathrm{s}
$$

Now, because $\alpha$ is spinning, we can compute the maximum velocity that a particle or spacecraft sitting on $\alpha$ would need to be able to reach the outer region of the system. In the $\alpha$ frame, its tangential velocity is given by $\left(\omega_{\alpha}-\omega\right) r_{c}=v_{s}$. With the $1999 \mathrm{KW} 4$ parameters, this gives $v_{s}=4.1 \times 10^{-4} \mathrm{~km} / \mathrm{s}$. Looking again at a location between the bodies, for a spacecraft leaving at $90 \mathrm{deg}$ from the surface of $\alpha$ at $v_{\max }$, the change in velocity needed is computed by

$$
\Delta v=\sqrt{\left(v_{s}^{2}+v_{\max \alpha}^{2}\right)}
$$

In this case, we find $\Delta v=9.6 \times 10^{-4} \mathrm{~km} / \mathrm{s}$, oriented at $155 \mathrm{deg}$ from the positive $x$ axis. On the other hand, if the velocity applied is along the tangential velocity coming from the primary spin, we would need

$$
\Delta v=v_{\max \alpha}-v_{s}
$$

For the current parameters of 1999 KW4, we obtain $\Delta v=4.6 \times 10^{-4} \mathrm{~km} / \mathrm{s}$, tangential to the surface.

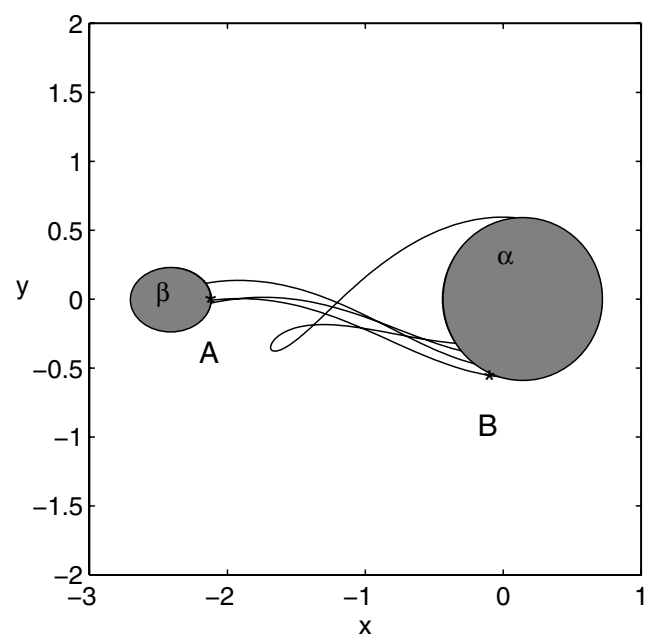

Fig. 15 Transit and nontransit trajectories for 1999 KW4.

\section{B. Velocity Constraints for Transit}

In Sec. $\underline{V}$, we investigated the conditions on the position and velocity for transit and nontransit trajectories. This is possible if $L_{1}$ is located outside of the ellipsoid. We retrieve the same situation for the binary 1999 KW4. Because the trajectories are governed by the binary gravity field, a spacecraft could just apply a small impulse and "jump" to coast to the other body.

Provided that the Jacobi value is higher than $C_{1}$, we computed the transit and nontransit trajectories as outlined in Sec. V. The conditions for nontransit trajectories are shown on Fig. $\overline{14}$. One example of nontransit trajectory and three transit trajectories are also shown on Fig. 15. Note that the black lines are the bodies themselves. We omitted the arrows giving the transit conditions on Fig. 15 for better clarity in reading the plot. For this binary system, we notice that most of the transit trajectories impact the surface of $\alpha$ on its outer regions. On $\beta$, the velocities are of the order of $v_{T \beta}=$ $1.80 \times 10^{-4} \mathrm{~km} / \mathrm{s}$, which is close to the velocity opening the outer region of the system, $v_{\max \beta}$, computed in the previous section. For $\alpha$, such transit velocity is about $v_{T \alpha}=5.75 \times 10^{-4} \mathrm{~km} / \mathrm{s}$, which is almost half of its $v_{\max \alpha}$. We can also notice that the system can be very sensitive to initial conditions, as some transit and nontransit trajectories have very close values of positions and velocities on $\alpha$.

Now, taking into account the spin rate of $\alpha$, we can compute the required launch or arrival velocities involved in transit trajectories. The situation of having a spacecraft leave $\beta$, in transit to $\alpha$, might be more appealing. For the spacecraft leaving $\alpha$, its spin rate makes the launch window on $\alpha$ small and periodic in time as opposed to launching from $\beta$, in which the launching location is fixed in time. As $\alpha$ is spinning, the relative position of the spacecraft in the RF3BP would need to be monitored so that transition to $\beta$ is possible. On the other hand, the spacecraft can transit from $\beta$ at any position on its surface, facing $\alpha$, provided it has the required velocity for transfer. Arriving on the spinning primary $\alpha$, simple geometry for the velocities would give the required velocity change to maintain the energy of the spacecraft below $v_{\max \alpha}$ and limit the possibility to reach the outer regions of the system and eventually escape.

To give a concrete example, we follow a spacecraft leaving $\beta$ at the point $A$ and arriving at $B$ on $\alpha$, shown on Fig. 15 . To transit to $\alpha$, we need $v_{A}=1.88 \times 10^{-4} \mathrm{~km} / \mathrm{s}$ oriented at $9.6 \overline{\mathrm{deg}}$ from the $x$ axis. Arriving at $\alpha$, the spacecraft velocity at $B$ would be $v_{B}=1.6 \times 10^{-4} \mathrm{~km} / \mathrm{s}$, at $12.4 \mathrm{deg}$ from the $x$ axis. Because $\alpha$ is spinning along the same direction, the total spacecraft velocity at $B$ would be

$$
v_{B \text { Total }}=v_{B}+v_{s}=5.7 \times 10^{-4} \mathrm{~km} / \mathrm{s}
$$

Because $v_{B \text { Total }}$ is lower than the velocity necessary to reach the outer region, found to be

$$
v_{\max \alpha}=8.7 \times 10^{-4} \mathrm{~km} / \mathrm{s}
$$

a spacecraft would not be able to escape from the system in this case.

\section{Conclusions}

In this work, we investigated the equilibrium solutions of the restricted full three-body problem in more detail. Taking the mass distribution of one of the bodies into account provides an additional physical constraint on the system. We first computed the conditions for the Lagrangian point $L_{1}$ to be sitting on the ellipsoid body, facing the sphere. This provides a limiting case of the binary system. Indeed, depending on the free parameters of the system, $L_{1}$ can be inside or outside of the ellipsoid.

For the case in which $L_{1}$ is between the two bodies, we looked at the corresponding linearized system. From investigating the manifold structure at $L_{1}$, we find conditions on a particle that may or may not allow transitional trajectories. We then map these conditions on the surface of the binary components to come up with initial and final conditions allowing transit and nontransit trajectories. We applied these methods to the binary system 1999 KW4 to come up with possible mission design. 
As rover exploration becomes more interesting and useful in sending spacecraft to small bodies, future work involves mission design and trajectory investigation for surface motion. This work showed that a vehicle approach by the small, secondary, ellipsoid body could benefit from the natural environment of a binary. In this case, with a velocity low enough, a spacecraft could orbit the binary system while staying close to the bodies. Then, using transit trajectories, a vehicle on the surface could easily transfer to the other body from a simple jump, as long as the transit conditions are satisfied. Because a vehicle would need to keep a low enough energy to stay close to the bodies, orbital and attitude dynamics couplings need to be studied; at impact on the other spinning body, for example, the geometry of a vehicle could make it gain momentum and open up outer regions of allowable motion of the binary system.

\section{Appendix: Derivatives of the Ellipsoid Potential}

Originally derived using Ivory's theorem [11], the potential energy expression for an ellipsoid, given the normalization mentioned, is written as

$$
\begin{gathered}
U_{e}=\frac{3}{4} \int_{\lambda}^{\infty} \phi(\boldsymbol{r}, v) \frac{\mathrm{d} v}{\Delta(v)} \\
\phi(\boldsymbol{r}, v)=1-\frac{x^{2}}{v+1}-\frac{y^{2}}{v+\beta^{2}}-\frac{z^{2}}{v+\gamma^{2}} \\
\Delta(v)=\sqrt{(v+1)\left(v+\beta^{2}\right)\left(v+\gamma^{2}\right)}
\end{gathered}
$$

where $0<\gamma \leq \beta \leq 1$ and $\lambda$ satisfies $\phi(r, \lambda)=0$. The $x$ axis is aligned with the longest axis of the ellipsoid, whereas the $z$ axis is along its shortest axis. The first derivatives of the ellipsoid potential are then given by [1]],

$$
\begin{aligned}
& U_{x}=-\frac{3}{2} x \int_{\lambda}^{\infty} \frac{\mathrm{d} u}{(u+1) \Delta(u)} \\
& U_{y}=-\frac{3}{2} y \int_{\lambda}^{\infty} \frac{\mathrm{d} u}{\left(u+\beta^{2}\right) \Delta(u)} \\
& U_{z}=-\frac{3}{2} z \int_{\lambda}^{\infty} \frac{\mathrm{d} u}{\left(u+\gamma^{2}\right) \Delta(u)}
\end{aligned}
$$

The derivatives are written in terms of the $R_{j}$ expressions that are the elliptic integrals representing the mass distribution of the ellipsoid. Using the substitution $v=u+\lambda$, they can be solved as written in the following form and can be computed using algorithms from [12]:

$$
\begin{aligned}
R_{j \alpha} & =\frac{3}{2} \int_{0}^{\infty} \frac{\mathrm{d} u}{(u+\lambda+1) \Delta(u+\lambda)} \\
R_{j \beta} & =\frac{3}{2} \int_{0}^{\infty} \frac{\mathrm{d} u}{\left(u+\lambda+\beta^{2}\right) \Delta(u+\lambda)}
\end{aligned}
$$

$$
R_{j \gamma}=\frac{3}{2} \int_{0}^{\infty} \frac{\mathrm{d} u}{\left(u+\lambda+\gamma^{2}\right) \Delta(u+\lambda)}
$$

\section{Acknowledgments}

This research was funded, in part, by a grant from the Jet Propulsion Laboratory/California Institute of Technology Director's Research and Development Fund. The authors also thank the Natural Sciences and Engineering Research Council of Canada.

\section{References}

[1] Scheeres, D. J., "Stability of Relative Equilibria in the Full Two-Body Problem," Annals of the New York Academy of Sciences, Vol. 1017, No. 1, 2004, pp. 81-94. doi:10.1196/annals.1311.006

[2] Scheeres, D. J., "Relative Equilibria for General Gravity Fields in the Sphere-Restricted Full Two-Body Problem," Celestial Mechanics and Dynamical Astronomy, Vol. 94, No. 3, 2006, pp. 317-349. doi:10.1007/s10569-005-6182-2

[3] Scheeres, D. J., and Augenstein, S., "Spacecraft Motion About Binary Asteroids," AAS/AIAA Astrodynamics 2003, Advances in the Astronautical Sciences, Univelt, San Diego, 2003, pp. 9911010.

[4] Bellerose, J., and Scheeres, D. J., "Stability of Equilibrium Points in the Restricted Full Three-Body Problem," Acta Astronautica, Vol. 60, No. 3, 2007, pp. 141-152. doi:10.1016/j.actaastro.2006.07.009

[5] Scheeres, D. J., Fahnestock, E. G., Ostro, S. J., Margot, J. -L., Benner, L. A. M., Broschart, S. B., Bellerose, J., Giorgini, J. D., Nolan, M. C., Magri, C., Pravec, P., Scheirich, P., Rose, R., Jurgens, R. F., Suzuki, S., and Dejong, E. M., "Dynamical Configuration of Binary Near-Earth Asteroid (66391) 1999 KW4," Science, Vol. 314, No. 1280, 2006, pp. $1280-1283$. doi:10.1126/science.1133599

[6] Kawaguchi, J., Kuninaka, H., Fujiwara, A., and Uesugi, T., "MUSESC, Its Launch and Early Orbit Operation," Acta Astronautica, Vol. 59, Nos. 8-11, 2006, pp. 669-678. doi:10.1016/j.actaastro.2005.07.002

[7] Danby, J. M. A., Fundamentals of Celestial Mechanics, 2nd ed., Willmann-Bell, Richmond, VA, 1992, pp. 102-111.

[8] Bellerose, J., and Scheeres, D. J., "Periodic Orbits in the Full Two-Body Problem," IAC Paper 06-C1.5.05, 2006.

[9] Conley, C. C., "Low Energy Transit Orbits in the Restricted ThreeBody Problem," SIAM Journal on Applied Mathematics, Vol. 16, No. 4, July 1968 , pp. 732-746. doi: $10.1137 / 0116060$

[10] Yoshimitsu, T., Kubota, T., Nakatani, I., Adachi, T., and Saito, H., "Micro-Hopping Robot for Asteroid Exploration," Acta Astronautica, Vol. 52, Nos. 2-6, 2003, pp. 441-446. doi:10.1016/S0094-5765(02)00186-8

[11] Moulton, F. R., An Introduction to Celestial Mechanics, Dover, New York, 1970, pp. 32-37.

[12] Flannery, B. P., Press, W. H., Teukolsky, S. A., and Vetterling, W. T., Numerical Recipes in C, The Art of Scientific Computing, 2nd ed., Cambridge Univ. Press, New York, 1996, pp. 254-263. 